\title{
Game Information Dynamics
}

\author{
Hiroyuki Iida and Takeo Nakagawa \\ Japan Advanced Institute of Science and Technology \\ 1-1 Asahidai, Nomi, Ishikawa, 923-1292, Japan \\ \{iida, takeo-n\}@jaist.ac.jp
}

\begin{abstract}
This paper is concerned with game information dynamics. Information and information kinetic energy are derived using existing models. Based on Einstein's special relativity theory, it is suggested that when the velocity of information particles is slightly smaller than the speed of light, our mental concentration increases enormously.
\end{abstract}

Keywords: Information Dynamics, Energy, Relativity Theory, Emotion.

\section{Introduction}

A central problem in game research has been to obtain the relationship between information of game outcome and game length (or time), e.g. [4, 5]. Here information of game outcome represents the data, which is the certainty of the game outcome. A natural extension is to obtain, e.g., information velocity and information acceleration. Recently, game information dynamic models have been proposed based on fluid mechanics ([2]). In the models, information is mathematically expressed as an analytical function of the game length (or time).

The two models are expressed, respectively, by

$$
\text { Model 1: } \xi=\eta^{\mathrm{n}} \text {, }
$$

and

$$
\text { Model 2: } \xi=[\sin (\pi / 2 \cdot \eta)]^{\mathrm{n}} \text {, }
$$

where $\xi$ is non-dimensional information of game outcome, $\eta$ non-dimensional game length (or time), and $\mathrm{n}$ a positive real number parameter. The value of the parameter $\mathrm{n}$ might depend on fairness of the game, strength of the two opponents, and strength difference between the two opponents. Information dynamics may be discussed using these models. Information kinetic energy, for example, can be obtained by using Model 1 or Model 2, following the definition in physics. It is considered that game information is closely related to emotions of observers or players ([3]).

The main purpose of the present paper is to clarify how information and information kinetic energy depend on game length, using previously proposed game information dynamic models ([2]). 


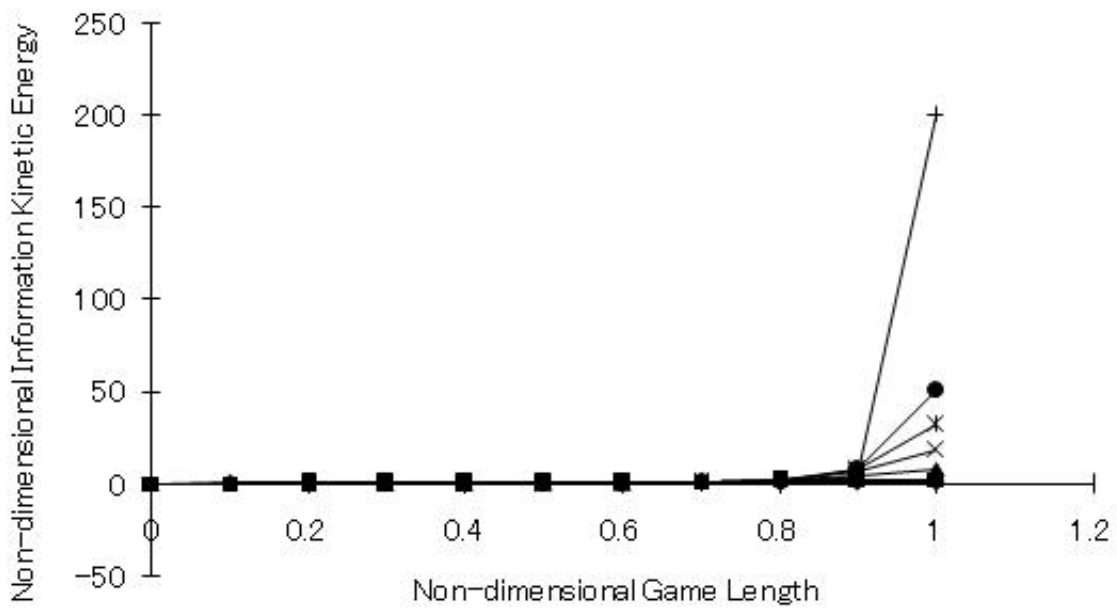

$\rightarrow_{n=1} \rightarrow{ }_{n=2} \rightarrow \leftarrow_{n=4} \quad \star_{n=6} \quad *_{n=8} \quad \rightarrow_{n=10} \quad 十_{n=20}$

Fig. 1. Non-dimensional information kinetic energy $E_{k}$ against non-dimensional game length $\eta$ for Model 1

\section{Information Dynamics}

Game information dynamics are, e.g., information, information momentum, information force and information energy, in relation to game length or time during a game. If information particles have mass in our brain (or informatical world) and flow exactly the same as fluid particles in the physical world, it is possible to discuss information dynamics. We have observed fair similarities between information momentum, information force and information kinetic energy with respect to game length. Therefore, in the present study we consider only information kinetic energy. We assume that information particles flow as electromagnetic particles (photons) or electrochemical particles in the informatical world and form a homogeneous information fluid.

Since Model 1 is expressed by (1), non-dimensional information velocity (first derivative) is

$$
\mathrm{d} \xi / \mathrm{d} \eta=\mathrm{n} \eta^{\mathrm{n}-1} \text {. }
$$

Since Model 2 is expressed by (2) the non-dimensional information velocity is derived as

$$
\mathrm{d} \xi / \mathrm{d} \eta=\mathrm{n} \cdot \pi / 2[\sin (\pi / 2 \cdot \eta)]^{\mathrm{n}-1} \cdot \cos (\pi / 2 \cdot \eta)
$$

Non-dimensional information kinetic energy $E_{k}$ is defined as 

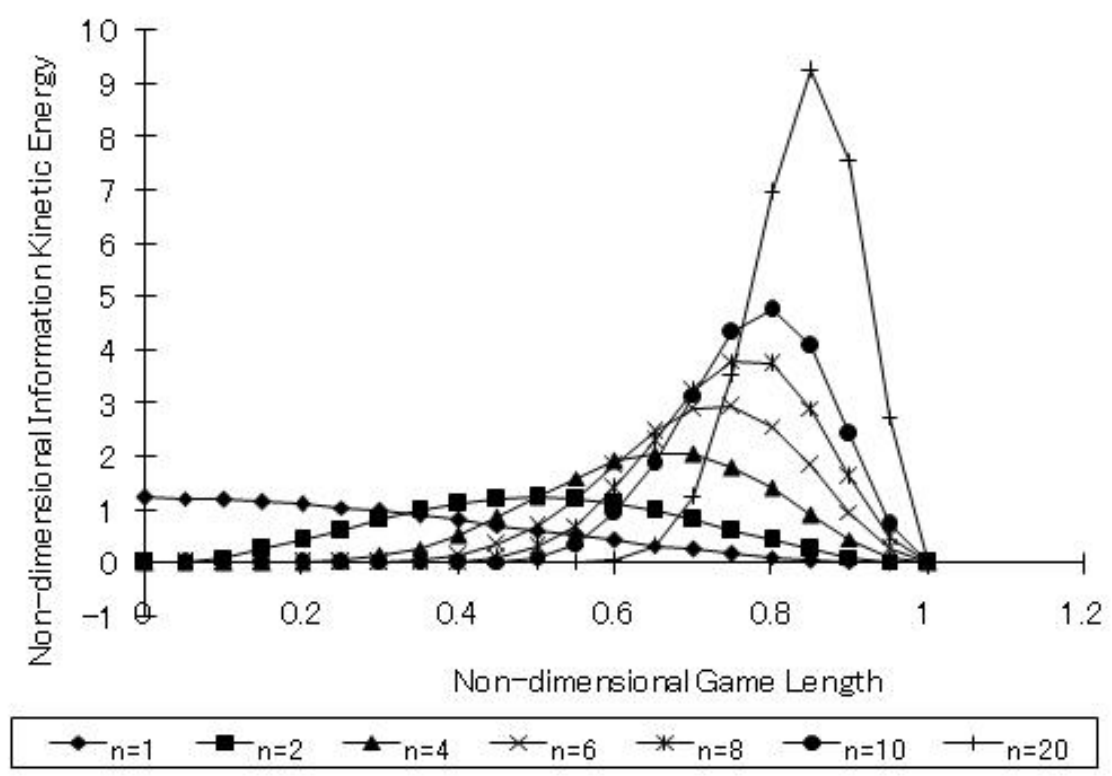

Fig. 2. Non-dimensional information kinetic energy $E_{k}$ against non-dimensional game length $\eta$ for Model 2

$$
\mathrm{E}_{\mathrm{k}}=1 / 2 \cdot \varphi \cdot(\mathrm{d} \xi / \mathrm{d \eta})^{2}
$$

Under the assumption of a homogeneous information fluid, non-dimensional information mass $\varphi=1$, and thus (5) becomes

$$
\mathrm{E}_{\mathrm{k}}=1 / 2 \cdot(\mathrm{d} \xi / \mathrm{d \eta})^{2} .
$$

This particular case has been considered. It is possible to calculate the value of $\mathrm{E}_{\mathrm{k}}$ by substituting either (3) or (4) into (6).

Figure 1 shows how non-dimensional information kinetic energy depends on nondimensional game length $\eta$ for Model 1.

Figure 2 shows how non-dimensional information kinetic energy $E_{k}$ depends on nondimensional game length $\eta$ for Model 2. When $n \geq 2, E_{k}$ increases with increasing $\eta$, takes a peak value and becomes zero at the end. However, the larger the value of $n$, the greater the peak value of $E_{k}$ as well as the value of $\eta$ at which $E_{k}$ takes the peak value. When $\mathrm{n} \leq 1, \mathrm{E}_{\mathrm{k}}$ decreases from an initial positive value to zero with increasing $\eta$.

\section{Discussion and Conclusion}

The slowing of time in a moving inertial frame with constant velocity $\mathrm{v}$, has been derived by Einstein([1]) as follows, 


$$
\Delta \mathrm{t}=\Delta \mathrm{t}_{0}\left[1-(\mathrm{v} / \mathrm{c})^{2}\right]^{1 / 2},
$$

where $\Delta \mathrm{t}$ is the elapsed time on the first inertial frame with velocity $\mathrm{v}$, as seen by an observer on the second inertial frame, and $\Delta \mathrm{t}_{0}$ the elapsed time for the observer on the second inertial frame. This denotes that the greater the velocity $\mathrm{v}$ is, the slower time proceeds on the first inertial frame, and vice versa.

Let us consider the case that the information particles (the first inertial frame) move with a constant velocity $\mathrm{v}$, relative to our body (the second inertial frame) fixed to the earth. Assuming again that motion of information particles reflects our emotions, when $\mathrm{v} \leq \mathrm{c}$, we may feel as if time stops, for the elapsed time on the information particles, corresponding to the first inertial frame with velocity $v$, becomes nearly equal to zero, as to be detected by the observer on the second inertial frame. For example, when an event proceeds on the first frame with constant velocity $\mathrm{v}$, the elapsed time on the first inertial frame is shorter than that on the second inertial frame. This means that the time on the first inertial frame proceeds slower than that on the second inertial frame, so that our cognitive sensor fixed to our body (the second inertial frame) perceives the event, being compacted within a shorter time. This may be the reason why our mental concentration increases with increasing information particle velocity $\mathrm{v}$. It is inferred that when $\mathrm{v} \leq \mathrm{c}$, our mental concentration increases enormously, and thus we can possess very high potential to overcome any problem or difficulty. Under this situation, a player equipped with high mental concentration in games such as Chess, Shogi, Go, Base Ball or Soccer can choose the best move or play.

\section{References}

1. Einstein, A.: Zur Elektrodynamik bewegter Korper. Annalen der Physik 17, 891-921 (1905)

2. Iida, H., Nakagawa, T., Spoerer, K.: A novel game information dynamic model based on fluid mechanics: case study using base ball data in world series 2010. In: Proceedings of the 2nd International Multi-Conference on Complexity Informatics and Cybernetics, pp. 134139 (2011)

3. Iida, H., Takehara, K., Nagashima, J., Kajihara, Y., Hashimoto, T.: An application of game refinement theory to mah-jong. In: International Conference on Entertainment Computing, pp. 333-338 (2004)

4. Majek, P., Iida, H.: Uncertainty of game outcome. In: 3rd International Conference on Global Research and Education in Intelligent Systems, pp. 171-180 (2004)

5. Iida, H., Nakagawa, T.: On Game Information Dynamics with reference to Soccer. Internal Technical Report No. 2011-06-21. Research Unit for Entertainment and Intelligence. Japan Advanced Institute of Science and Technology (2011) 